TẠP CHÍ KHOA HỌC ĐẠI HỌC TÂN TRÀO
ISSN: 2354 - 1431

\title{
KHAI THÁC CÁC TÍNH CHẤT SỐ HỌC LIÊN QUAN ĐẾN BÀI TOÁN VỀ DÃY SỐ TRONG CÁC KÌ THI OLYMPIC SINH VIÊN
}

\author{
Dương Thị Hồng Hảia, Lê Thiếu Tráng ${ }^{a, *}$ \\ ${ }^{a}$ Truòng Đại học Tân Trào \\ "Email: lttrang0466@tuyenquang.edu.vn
}

\section{Thông tin bài viết}

Ngày nhận bài:

$2 / 5 / 2020$

Ngày duyệt đăng:

$10 / 6 / 2020$

Tù khóa:

Dãy số, giới hạn, số học, số chính phwơng, sinh viên.

\section{Tóm tắt}

Dãy số là một trong những chủ đề nằm trong chương trình giải tích của chương trình Đại học Sư phạm Toán, là một chuyên đề cơ bản trong nội dung dạy học cho các đội tuyển Olympic dành cho sinh viên toán các trường Đại học và Cao đẳng. Bài toán về dãy số giúp sinh viên hiểu sâu sắc hơn về hàm số, về qui luật phân bố các số, về tính chất các vô cùng bé, vô cùng lớn... Bài viết này, tác giả tổng hợp một số bài toán liên quan đến dãy số nhằm phát triển năng lực học tập và nghiên cứu của sinh viên.

\section{1. ĐĀT VẤN ĐỀ}

Trong bài báo này, tác giả dựa trên các ý tưởng đã có về số học và dãy số của một số tác giả như GS.TS Phan Huy Khải (Viện Toán học Việt Nam), một số bài toán trong Tạp chí Toán Học và Tuổi trẻ và một số chuyên đề về dãy số, làm sáng tỏ một số vấn đề học sinh và sinh viên còn chưa rõ khi giải toán dạng này, hình thành phương pháp chung giải các dạng toán đó.

Thực tế giảng dạy, sinh viên tham gia dự thi Olympic tại Trường Đại học Tân Trào năm 2018- 2019 tác giả nhận thấy, sinh viên đội tuyển toán chưa nắm được hệ thống các ứng dụng số học vào dãy số hiệu quả, chưa có cái nhìn tổng thể, nguồn gốc các bài toán và chưa có tính chủ động, sáng tạo trong thực tiễn. Do đó, để các đội tuyển sinh viên đạt kết quả cao trong các kì thi Olympic cần thiết phải trang bị cho các em các kiến thức về dãy số thông qua một số bài toán cơ bản.

\section{NÔিI DUNG NGHIÊN CÚU}

Trong phần nội dung, do chuyên đề này là ứng dụng số học vào dãy số, nên kiến thức cơ sở sẽ được đề cập trong từng bài cụ thể.

2.1. Bài toán 1. Tính tổng

$\mathrm{S}_{\mathrm{k}}=1^{\mathrm{k}}+2^{\mathrm{k}}+\ldots+\mathrm{n}^{\mathrm{k}}, \mathrm{n} \in ¥ *, \mathrm{k} \in ¥$.

2.1.1. Xây dựng công thức tính:

Ta đã biết:

$$
\begin{aligned}
& \mathrm{S}_{0}=1^{0}+2^{0}+\ldots+\mathrm{n}^{0}=\mathrm{n} \\
& \mathrm{S}_{1}=1^{1}+2^{1}+\ldots+\mathrm{n}^{1}=\frac{\mathrm{n}(\mathrm{n}+1)}{2}
\end{aligned}
$$




$$
S_{2}=1^{2}+2^{2}+\ldots+n^{2}=\frac{n(n+1)(2 n+1)}{6}
$$

Đã có nhiều cách để tính các tổng trên, nhưng để tổng quát được, ta có thể làm như sau:

Ta đã có công thức: $(a+b)^{n}=\sum_{k=0}^{n} C_{n} a^{n-k} b^{k}$.

Áp dụng vào các khai triển sau:

$$
(\mathrm{n}+1)^{2}-1=\mathrm{C}_{2} \mathrm{~S}_{1}+\mathrm{C}_{2}^{2} \mathrm{~S}_{0}
$$$$
(\mathrm{n}+1)^{3}-1=(\mathrm{n}+1)^{3}-1=\mathrm{C}_{3} \mathrm{~S}_{2}+\mathrm{C}_{3}^{2} \mathrm{~S}_{1}+\mathrm{C}_{3}^{3} \mathrm{~S}_{0} \ldots
$$

Tổng quát ta có công thức truy hồi cho $\mathrm{S}_{\mathrm{K}}$ :

$$
(\mathrm{n}+1)^{\mathrm{k}}-1=\mathrm{C}_{\mathrm{k}+1} \mathrm{~S}_{\mathrm{k}}+\mathrm{C}_{\mathrm{k}+1}^{2} \mathrm{~S}_{\mathrm{k}-1}+\ldots+\mathrm{C}_{\mathrm{k}+1}^{\mathrm{k}} \mathrm{S}_{1}+\mathrm{C}_{\mathrm{k}+1}^{\mathrm{k}+1} \mathrm{~S}_{0}
$$

2.1.2. Ví dụ 1. Tìm số hạng tổng quát của dãy số $\left(\mathrm{u}_{\mathrm{n}}\right)$, biết:

$$
\mathrm{u}_{1}=0, \mathrm{u}_{\mathrm{n}+1}=\frac{\mathrm{n}(\mathrm{n}+1)}{(\mathrm{n}+2)(\mathrm{n}+3)}\left(\mathrm{u}_{\mathrm{n}}+1\right), \mathrm{n} \in ¥ * .
$$

Giải: Phương trình dãy được viết lại: $\mathrm{u}_{\mathrm{n}+1}=$ $u_{n+1}=\frac{n(n+1)^{2}(n+2)}{(n+1)(n+2)^{2}(n+3)}\left(u_{n}+1\right)$

$$
\begin{gathered}
\Leftrightarrow(n+1)(n+2)^{2}(n+3) u_{n+1}=n(n+1)^{2}(n+2) u_{n}+n(n+1)^{2}(n+2) . \\
\text { Đặt } n(n+1)^{2}(n+2) u_{n}=x_{n} \\
\text { và } n(n+1)^{2}(n+2)=f_{n} \text {, ta có phương trình: } \\
x_{n+1}-x_{n}=f_{n}, x_{1}=0 .
\end{gathered}
$$

Cho $\mathrm{n}=1,2, . .$. , ta có:

$$
\begin{aligned}
& x_{n}-x_{n-1}=(n-1) \cdot n^{2} \cdot(n+1) \\
& x_{n-1}-x_{x-2}=(n-2) \cdot(n-1)^{2} \cdot n \ldots \\
& x_{2}-x_{1}=1.2^{2} \cdot 3
\end{aligned}
$$

Cộng các đẳng thức và rút gọn ta được:

$$
x_{n}-x_{1}=1 \cdot 2 \cdot 3+23 \cdot 4+\ldots+(n-1) \cdot n^{2} \cdot(n+1) .
$$

Thay $\mathrm{X}_{1}=0$ ta có:

$$
x_{n}=1 \cdot 2 \cdot 3+2 \cdot 3 \cdot 4+\ldots+(n-1) \cdot n^{2} \cdot(n+1) \text {. }
$$

Ta hãy tìm cách tính tổng trên, ta có:

$$
(n-1) \cdot n^{2} \cdot(n+1)=n^{4}-n^{2} \text {. }
$$

Do đó:

$$
x_{n}=\left(1^{4}+2^{4}+\ldots+n^{4}\right)-\left(1^{2}+2^{2}+\ldots+n^{2}\right)
$$

Đây chính là tổng $S_{4}$ và $S_{2}$ ở trên. Thay vào ta được:

$$
\begin{aligned}
& x_{n}=\frac{(n-1) n(n+1)(2 n+1)(n+2)}{10} \\
& \Rightarrow u_{n}=\frac{(n-1)(2 n+1)}{10(n+1)}, n \in ¥ * .
\end{aligned}
$$

Nhận xét: Bài toán trên là loại dãy sai phân tuyến tính với hệ số biến thiên: Tìm số hạng tổng quát của dãy $\left(u_{n}\right)$ biết $u_{1}=a ; u_{n+1}=\frac{n(n+1) . .(n+k)}{(n+k+1) . .(n+2 k+1)}\left(u_{n}+1\right), n \in ¥ *$.

\subsubsection{Bài tập tương tự}

Bài 1.1. Cho dãy số $\left(\mathrm{u}_{\mathrm{n}}\right)$ biết: $u_{n}=\frac{21^{2}+3 \cdot 2^{2}+\ldots+(n+1) \cdot n^{2}}{n^{4}}$. Tính $\operatorname{limu}_{n}$ ?

Bài 1.2. Tính các giới hạn sau:

1) $\lim \frac{1^{5}+2^{5}+\ldots+n^{5}}{n^{6}}$;

2) $\lim ^{\mathrm{k}^{\mathrm{k}}+2^{\mathrm{k}}+\ldots+\mathrm{n}^{\mathrm{k}}} \mathrm{n}^{\mathrm{k}+1}$.

Bài 1.3. Tìm $\mathbf{U}_{n}$ biết

$$
u_{1}=1, u_{n+1}=\frac{n+1}{n+2}\left(u_{n}+2\right), n \in ¥ * .
$$

2.2. Bài toán 2. Tìm điều kiện hoặc chứng minh các số hạng của một dãy số không đổi dấu.

2.2.1. Ví dụ: Cho dãy $\left(a_{n}\right)$ biết $a_{0}=a, a_{1}=b$ và $a_{n+2}=-a_{n+1}+2 a_{n}+6$. Tìm điều kiện của $a, b$ để $a_{n}>0, \forall n \in ¥$.

Giải:

+ Tìm số hạng tổng quát của dãy: Đặt $a_{n}=V_{n}+W_{n}$, với $\left(V_{n}\right)$ là dãy tuyến tính thuần nhất, $\left(\mathrm{W}_{\mathrm{n}}\right)$ là dãy đa thức của $\mathbf{n}$. Phương trình đặc trưng của dãy là $\mathrm{X}^{2}+\mathrm{X}-2=0$, có nghiệm $\mathrm{x}_{1}=1, \mathrm{x}_{2}=-2$, nên ta có:

$$
\begin{aligned}
& \quad \mathrm{v}_{\mathrm{n}}=\mathrm{A} 1^{\mathrm{n}}+\mathrm{B}(-2)^{\mathrm{n}}=\mathrm{A}+\mathrm{B}(-2)^{\mathrm{n}} \text { và } \\
& \mathrm{w}_{\mathrm{n}}=\mathrm{Cn} .
\end{aligned}
$$

Thay vào dãy ta có:

$$
\mathrm{C}(\mathrm{n}+2)=-\mathrm{C}(\mathrm{n}+1)+2 \mathrm{Cn}+6 \Rightarrow \mathrm{C}=2
$$


Do đó: $\mathrm{W}_{\mathrm{n}}=2 \mathrm{n}$. Vậy:

$$
a_{n}=A+B(-2)^{n}+2 n, n \in ¥ \text {. }
$$

Thay $\mathrm{n}=0$, l ta có:

$$
a_{n}=(-2)^{n}\left[\frac{2 a+b-2}{(-2)^{n}}+\frac{2 n}{(-2)^{n}}+\frac{a-b+2}{3}\right\rfloor, n \in ¥ .
$$

Từ đó ta thấy:

- Nếu $\frac{a-b+2}{3}>0$ thì với $n=2 k+1$ có $\operatorname{lima}_{n}=-\propto$, loại.

- Nếu $\frac{a-b+2}{3}<0$ thì với $n=2 k$ có $\lim _{\mathrm{n}}=-\propto$, loại.

$\Rightarrow \frac{a-b+2}{3}=0$ thoả mãn và điều kiện cần tìm của

$a, b$ là $b=a+2$ và $a>0$.

\subsubsection{Bài tập tương tự}

Bài 2.1. Xác định các dãy $\left(\mathrm{a}_{\mathrm{n}}\right)$ biết: $a_{0}=1, a_{n+2}=-a_{n+1}+a_{n}$ mà $a_{n}>0, \forall n \in ¥$.

Bài 2.2. Chứng minh có duy nhất 1 dãy số dương $\left(u_{n}\right)$ thoả mãn điều kiện:

$$
u_{0}=1, u_{n}-u_{n+1}=u_{n+2}, \forall n \in ¥ .
$$

2.3. Bài toán 3. Lập luận để các số hạng của một dãy số là số nguyên.

2.3.1. Ví dụ: Cho dãy $\left(a_{n}\right)$ biết: $a_{1}=a_{2}=1$, $a_{n}=\frac{a_{n-1}^{2}+2}{a_{n-2}}, \forall n \geq 3$. Chứng minh mọi số hạng của dãy đều là số nguyên.

Giải: Trước hết ta đưa dãy về dạng tuyến tính. Ta có: $a_{3}=3, a_{4}=11, a_{5}=41$. Giả sử $a_{n}=O a_{n-1}+\beta a_{n-2}+\gamma$. Thay $n=1,2,3$ ta được hệ: $\left\{\begin{array}{l}3=\alpha+\beta+\gamma \\ 11=3 \alpha+\beta+\gamma \\ 41=11 \alpha+3 \beta+\gamma\end{array} \Leftrightarrow\left\{\begin{array}{l}\alpha=4 \\ \beta=-1 \\ \gamma=0\end{array}\right.\right.$.

Vậy: $a_{n}=4 a_{n-1}-a_{n-2}, n \geq 3$.

Do $\mathrm{a}_{1}=\mathrm{a}_{2}=1 \in \mathscr{\ell} \Rightarrow \mathrm{a}_{\mathrm{n}} \in \boldsymbol{\phi}, \forall n \in ¥ *$.

\subsubsection{Các bài tập tương tự}

Bài 3.1. Cho dãy $\left(\mathrm{X}_{\mathrm{n}}\right)$ biết:

$$
x_{1}=1, x_{n+1}=3 x_{n}+\sqrt{8 x_{n}^{2}+1}, n \in ¥ * .
$$

Chứng minh mọi số hạng của dãy đều nguyên.

Bài 3.2. Cho dãy $\left(u_{n}\right)$ thoả mãn:

$u_{n+2}=\frac{u_{n} \cdot u_{n+1}}{2 u_{n}-u_{n+1}}, \forall n=1,2, \ldots$ Tìm điều kiện cần và đủ đối với $\mathrm{U}_{1}, \mathrm{U}_{2}$ để dãy có vô số số hạng nguyên.

Bài 3.3. Cho dãy $\left(a_{n}\right)$ biết:

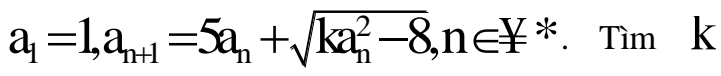
nguyên dương để mọi số hạng của dãy đều nguyên.

Bài 3.4. Bài toán tổng quát 1 : Cho $\mathrm{a}, \mathrm{b} \in \mathcal{L}^{\mathrm{v}}$ vã dãy $\left(a_{n}\right)$ biết:

$$
\begin{gathered}
\mathrm{a}_{1}=1, \mathrm{a}_{\mathrm{n}+1}=\mathrm{a} \mathrm{a}_{\mathrm{n}}+\sqrt{\mathrm{k} \cdot \mathrm{a}_{\mathrm{n}}^{2}-\mathrm{b}} . \text { Tìm } \mathrm{k} \text { nguyên } \\
\text { dương để } \mathrm{a}_{\mathrm{n}} \in \boldsymbol{\phi}, \forall \mathrm{n} \in \supsetneqq *
\end{gathered}
$$

Bài 3.5. Bài toán tổng quát 2: Cho các số nguyên $a, b, c$ thoả mãn: $a^{2}=b+1$. Dãy $\left(u_{n}\right)$ được xác định như sau:

$\mathrm{u}_{0}=0, \mathrm{u}_{\mathrm{n}+1}=\mathrm{au}_{\mathrm{n}}+\sqrt{\mathrm{bu}_{\mathrm{n}}^{2}+\mathrm{c}^{2}}, \mathrm{n} \in ¥$. Chứng mọi số hạng của dãy đều nguyên.

Bài 3.6. Chứng minh tồn tại đúng một dãy $\left(u_{n}\right)$ nguyên thoả mãn:

$$
\mathrm{u}_{1}=1, \mathrm{u}_{2}>1, \mathrm{u}_{1}=1, \mathrm{u}_{2}>1, \mathrm{u}_{\mathrm{n}+1}^{3}+1=\mathrm{u}_{\mathrm{n}} \mathrm{u}_{\mathrm{n}+2}, \forall n=1,2, \ldots
$$

2.4. Bài toán 4. Chứng minh, phát hiện các đẳng thức về dãy số, số chính phương và số lập phương.

2.4.1. Ví dụ: Cho dãy $\left(a_{n}\right)$ với

$$
a_{0}=1, a_{1}=13, a_{n+2}=14 a_{n+1}-a_{n}, n \in ¥ \text {. }
$$

Chứng minh với mọi số tự nhiên nta có:

1) $a_{n+1}^{2}-a_{n} \cdot a_{n+2}+12=0$.

2) $\frac{4 \mathrm{a}_{\mathrm{n}}^{2}-1}{3}$ là số chính phương. Từ đó suy ra số $2 \mathrm{a}_{\mathrm{n}}-1$ và $\frac{2 \mathrm{a}_{\mathrm{n}}+1}{3}$ cũng là các số chính phương.

3) $a_{n}$ là tổng của hai số chính phương liên tiếp và $a_{n}^{2}$ là hiệu của hai số lập phương liên tiếp. 


\section{Giải:}

1) Câu hỏi 1) có thể làm bằng phương pháp qui nạp (các tài liệu đều giải theo cách này). Nhưng vấn đề đặt ra là: Nếu không yêu cầu chứng minh đẳng thức trên, mà hỏi các câu hỏi sau thì học sinh sẽ xử lí như thế nào? Ta hãy hướng dẫn học sinh tự xây dựng được đẳng thức phần 1), đó cũng là cách khác để chứng minh đẳng thức này:

Từ giả thiết dễ thấy $\mathrm{a}_{\mathrm{n}} \neq 0, \forall n \in ¥$ và $\mathrm{a}_{2}=181$.

Nên đẳng thức:

$$
a_{n+2}=14 a_{n+1}-a_{n} \Leftrightarrow \frac{a_{n+2}+a_{n}}{a_{n+1}}=14 .
$$

Thay $n_{\text {bởi }} \mathrm{n}-\mathbf{l}$ ta được:

$$
\begin{aligned}
& \frac{a_{n+1}+a_{n-1}}{a_{n}}=14 \Rightarrow \frac{a_{n+2}+a_{n}}{a_{n+1}}=\frac{a_{n+1}+a_{n-1}}{a_{n}} \\
& \Leftrightarrow a_{n+1}^{2}-a_{n} \cdot a_{n+2}=a_{n}^{2}-a_{n-1} \cdot a_{n+1} . \text { Truy hồi biểu }
\end{aligned}
$$
thức trên ta được:

$$
a_{n+1}^{2}-a_{n} \cdot a_{n+2}=a_{1}^{2}-a_{0} \cdot a_{2}=-12=-12 .
$$

$a_{n+1}^{2}-a_{n} \cdot a_{n+2}+12=0, \forall \in ¥$.

2) Từ giả thiết dễ thấy $a_{n} \in \mathscr{\ell}, \forall n \in ¥$. Theo 1) thì phương trình: $a_{n+1}^{2}-a_{n} \cdot a_{n+2}+12=0$.

$$
\Leftrightarrow a_{n+1}^{2}-a_{n} \cdot\left(14 a_{n+1}-a_{n}\right)+12=0
$$

$\Leftrightarrow a_{n+1}^{2}-14 a_{n+1} \cdot a_{n}+a+12=0$ phải có nghiệm $\mathrm{X}=\mathrm{a}_{\mathrm{n}+1} \in \not \subset \Leftrightarrow \Delta^{\prime}=48 \mathrm{a}_{\mathrm{n}}^{2}-12$ phải là số chính phương.

Ta có $\Delta^{\prime}=36 .\left(\frac{4 a_{n}^{2}-1}{3}\right)$ chính phương $\Rightarrow\left(\frac{4 a_{n}^{2}-1}{3}\right)$ là số chính phương.

Ta cũng có: $\left(\frac{4 a_{n}^{2}-1}{3}\right)=\left(2 a_{n}-1\right)\left(\frac{2 a_{n}+1}{3}\right)$ chính phương.

Để hai số $2 \mathrm{a}_{\mathrm{n}}-1$ và $\frac{2 \mathrm{a}_{\mathrm{n}}+1}{3}$ chính phương thì phải có: $\left\{\begin{array}{l}\left(2 a_{n}-1, \frac{2 a_{n}+1}{3}\right)=1 \\ 2 a_{n}+11 B 1\end{array}\right.$
Điều này đúng vì $\left(2 \mathrm{a}_{\mathrm{n}}-1,2 \mathrm{a}_{\mathrm{n}}+1\right)=1$ nên $\left(2 \mathrm{a}_{\mathrm{n}}-1, \frac{2 \mathrm{a}_{\mathrm{n}}+1}{3}\right)=1$

Mặt khác $\mathrm{a}_{\mathrm{n}} \equiv 1(\bmod \mathcal{B})$ (chứng minh quy nạp) $\Rightarrow 2 \mathrm{a}_{\mathrm{n}}+3 \mathrm{~B}$.

3) Vì $2 a_{n}-1$ và $\frac{4 a_{n}^{2}-1}{3}$ là những số chính phương lẻ nên ta có:

$2 \mathrm{a}_{\mathrm{n}}-1=(2 \mathrm{k}+1)^{2} \Rightarrow \mathrm{a}_{\mathrm{n}}=2 \mathrm{k}^{2}+2 \mathrm{k}+1=\mathrm{k}^{2}+(\mathrm{k}+1)^{2}$. $\frac{4 \mathrm{a}_{\mathrm{n}}^{2}-1}{3}=(2 \mathrm{k}+1)^{2} \Rightarrow \mathrm{a}=3 \mathrm{k}^{2} 3 \mathrm{k}+1=(\mathrm{k}+1)^{3}-\mathrm{k}^{3}$.

2.4.2. Bài tập tương tự

Bài 4.1. Cho dãy số $\left(a_{n}\right)$ với

$a_{0}=2, a_{n+1}=4 a_{n}+\sqrt{15 a_{n}^{2}-60}, n \in ¥$. Tìm $a_{n}$ và chứng minh: $\frac{1}{5}\left(a_{2 n}+8\right)$ bằng tổng bình phương 3 số nguyên liên tiếp, $\forall n \in ¥ *$.

Bài 4.2. Cho dãy $\left(u_{n}\right)$ biết $u_{1}=1, u_{2}=3, u_{n+1}=(n+2) u_{n}-(n+1) u_{n-1}, n=23, \ldots$ Tìm $n$ để $\mathrm{U}_{\mathrm{n}}$ là số chính phương.

Bài 4.3. Cho dãy số nguyên $\left(\mathrm{u}_{\mathrm{n}}\right)$ với $\mathrm{u}_{0}=1, \mathrm{u}_{1}=45, \mathrm{u}_{\mathrm{n}+2}=45 \mathrm{u}_{\mathrm{n}+1}-7 \mathrm{u}_{\mathrm{n}}, \forall \in ¥$.

1) Tìm số ước số tự nhiên của số

$$
\mathrm{M}=\mathrm{u}_{\mathrm{n}+1}^{2}-\mathrm{u}_{\mathrm{n}} \cdot \mathrm{u}_{\mathrm{n}+2} \text {. }
$$

2) Chứng minh với mọi số tự nhiên $\mathrm{n}$ thì số $A_{n}=1997 .+4.7^{n+1}$ là số chính phương.

Bài 4.4. Cho dãy $\left(\mathrm{u}_{\mathrm{n}}\right)$ biết $\mathrm{u}_{0}=3, \mathrm{u}_{1}=17, \mathrm{u}_{\mathrm{n}}=6 \mathrm{u}_{\mathrm{n}-1}-\mathrm{u}_{\mathrm{n}-2}, \forall \mathrm{n}=2,3, \ldots$ Chứng minh $\forall n \in ¥$ thì $u_{n}^{2}-\mathbb{N} \mathbb{V}$ và thương là số chính phương.

Bài 4.5. Cho dãy $\left(u_{n}\right)$ biết

$$
u_{1}=1, u_{2}=-1, u_{n}=-u_{n-1}-2 u_{n-2}, n \geq 3 \text {. }
$$

Lập dãy $\left(\mathrm{V}_{\mathrm{n}}\right)$ với $\mathrm{v}_{\mathrm{n}}=2^{\mathrm{n}+1}-7 \mathrm{u}_{\mathrm{n}-1}^{2}, \mathrm{n}=2,3$,...Chứng minh mọi số hạng của dãy $\left(\mathrm{V}_{\mathrm{n}}\right)$ là số chính phương. 
2.5. Bài toán 5. Các bài toán liên quan đến tính chất chia hết và có dư

2.5.1. Ví dụ: Cho dãy $\left(u_{n}\right)$ biết:

$$
u_{n+2}=\left\{\begin{array}{l}
u_{0}=1, u_{1}=3 \text { và } \\
u_{n+1}+9 u_{n}: n=2 k \\
9 u_{n+1}+5 u_{n}: n=2 k+1
\end{array}, \forall n \in ¥ .\right.
$$

Tính tổng $S=\sum_{i=0}^{19} u_{i}^{2}$. Tìm số dư khi chia $S$ cho 8 , cho 5 và cho 40 .

Giải:

+ Từ giả thiết ta tính được:

$$
\begin{aligned}
& \left\{\begin{array}{l}
u_{2 n+2}=u_{2 n+1}+9 u_{2 n} \\
u_{2 n+1}=9 u_{2 n}+5 u_{2 n-1}
\end{array} \Rightarrow\right. \\
& \left\{\begin{array}{l}
u_{2 n+2} \equiv u_{2 n+1}+u_{2 n}(\bmod 4) \\
u_{2 n+1} \equiv u_{2 n}+u_{2 n-1}
\end{array}\right.
\end{aligned}
$$

Khai triển dãy: $\quad \mathrm{u}_{0} \quad \mathrm{u}_{1} \quad \mathrm{u}_{2}$

$\begin{array}{lcccc}\mathrm{u}_{3} & \mathrm{u}_{4} & \mathrm{u}_{5} \ldots . . & & \\ & & & 1 & 3 \\ 12 & 123 & 121 & 2794 & \\ \text { Do đó } & \mathrm{u}_{0} & \mathrm{u}_{1} & \mathrm{u}_{2} & \mathrm{u}_{3} \\ \mathrm{u}_{4} & \mathrm{u}_{5} & \mathrm{u}_{6} & \mathrm{u}_{7} & \mathrm{u}_{8} \\ \mathrm{u}_{9} & \ldots & \text { lần lượt đồng dư } & 1 & 3 \\ 0 & 3 & 3 & 2 & 1 \\ 3 & 0 & 3 & (\bmod 4) & \end{array}$

$\Rightarrow$ Dãy số dư tuần hoàn chu kỳ $6(\bmod 4), \forall n \geq 2$.

Vậy:

$\mathrm{S} \equiv 1^{2}+3^{2}+333\left(0^{2}+3^{2}+3^{2}+2^{2}+1^{2}+3^{2}\right)=10666 \equiv 2$

$(\bmod 4) \Rightarrow S \equiv 2(\bmod 8)$.

+ Tương tự trên ta có:

$\left\{\begin{array}{l}\mathrm{u}_{2 \mathrm{n}+2} \equiv \mathrm{u}_{2 \mathrm{n}+1}+\mathrm{u}_{2 \mathrm{n}}(\bmod 5) \text {. Khai triển được } \\ \mathrm{u}_{2 \mathrm{n}+1} \equiv \mathrm{u}_{2 \mathrm{n}}\end{array}\right.$

$\left(\mathrm{u}_{\mathrm{n}}\right)$ tuần hoàn chu kỳ $8(\bmod 5), \forall \mathrm{n} \geq 2$, nên

$\mathrm{S}=1^{2}+3^{2}+249\left(2^{2}+3^{2}+. .+1^{2}\right) \equiv 3(\bmod 5)$

+ Từ hai ý trên do $(8,5)=1$ nên ta có $S \equiv 18$ $(\bmod 40)$.

\subsubsection{Bài tập tương tự}

Bài 5.1. Cho dãy $\left(\mathrm{b}_{\mathrm{n}}\right)$ với

$b_{1}=0, b_{2}=14, b_{3}=-18, b_{n+1}=7 b_{n-1}-6 b_{n-2}, \forall n \in ¥ *$.

Chứng minh nếu plà số nguyên tố thì $\mathrm{b}_{\mathrm{p}} \mathrm{l} \mathbf{A}$.
Bài 5.2. a) Cho hai dãy $\left(u_{n}\right)$ và $\left(v_{n}\right)$ xác định như sau:

$$
\begin{aligned}
& \mathrm{u}_{0}=\mathrm{u}_{1}=1, \mathrm{u}_{\mathrm{n}+1}=\mathrm{u}_{\mathrm{n}}+2 \mathrm{u}_{\mathrm{n}-1}, \forall \mathrm{n}=1,2 . . \\
& \mathrm{v}_{0}=1, \mathrm{v}_{1}=7, \mathrm{v}_{\mathrm{n}+1}=2 \mathrm{v}_{\mathrm{n}}+3 \mathrm{v}_{\mathrm{n}-1}, \forall \mathrm{n}=1,2 . .
\end{aligned}
$$

Tìm $\mathrm{U}_{\mathrm{n}}$ và $\mathrm{V}_{\mathrm{n}}$ ?

Chứng minh trong 2 dãy trên chỉ có 1 hạng tử chung, ngoài ra không còn hạng tử nào chung khác.

Bài 5.3. Cho dãy $\left(\mathbf{u}_{n}\right)$ xác định như sau:

$$
\mathrm{u}_{\mathrm{n}}=\frac{(2+\sqrt{3})^{\mathrm{n}}-(2-\sqrt{3})^{\mathrm{n}}}{2 \sqrt{3}}, \forall \mathrm{n}=0,1,2, \ldots
$$

Chứng minh

$\mathrm{u}_{\mathrm{n}} \in \mathscr{\ell}, \forall \mathrm{n}=0,1,2$.. Tìm tất cả các số hạng của dãy chia hết cho 3 .

$$
\begin{aligned}
& \text { Bài 5.4. Cho dãy }\left(a_{n}\right) \text { với } \\
& a_{0}=19, a_{1}=98, a_{n+2}=a_{n}+a_{n+1} \cdot \\
& \text { Tìm số dư khi chia } S=\sum_{0}^{2} a_{i}^{2} \text { cho 8. }
\end{aligned}
$$

Bài 5.5. Cho dãy $\left(u_{n}\right), n \in ¥ *$ với

$$
u_{1}=2, u_{n}=3 u_{n-1}+2 n^{3}-9 n^{2}-3, n=2,3, \ldots
$$

Chứng minh với mỗi số p nguyên tố ta có:

$$
2000 \sum_{i=1}^{1} u_{i} 14
$$

\section{KẾT LUẬN}

Qua một số bài toán cơ bản nêu trên, sẽ góp phần giúp các em sinh viên rút ra được định hướng tư duy và phương pháp giải bài toán số học liên quan đến dãy số trong các kì thi Olympic Toán. Với những đánh giá và bổ sung về lí luận và phương pháp giải sẽ giúp sinh viên củng cố vững hơn về dạng toán và giải quyết những bài toán khó hơn. Tuy nhiên, bài toán về dãy số là một chủ đề rất rộng nên rất cần tiếp tục có nghiên cứu, tổng hợp chuyên sâu đến những dạng toán thường gặp trong các kì thi Olympic Toán của học sinh, sinh viên, đặc biệt là những bài toán giúp phát triển năng lực tư duy Toán học cho học sinh, sinh viên, góp phần nâng cao chất lượng dạy học môn toán theo hướng tập trung vào phát triển năng lực người học. 


\section{TÀI LIỆU THAM KHẢO}

[1]. Phan Huy Khải, Các chuyên đề số học bồi dương học sinh giỏi Toán-Chuyên đề Số học và dãy số, Nxb Giáo dục, 2016 (tái bản).

[2]. Bộ Giáo dục và Đào tạo - Hội Toán học Việt Nam, Tuyển tập 30 năm Tạp chí Toán học và Tuổi trẻ, Nxb Giáo dục, 2013 (tái bản).

[3] Nguyễn Xuân Liêm (1997), Giải tích tập 1, Nhà xuất bản Giáo dục, Hà Nội.
[4] Trần Đức Long - Nguyễn Đình Sang - Nguyễn Viết Triều Tiên - Hoàng Quốc Toàn (2008), Bài tập giải tích tập I, Nhà xuất bản ĐHQG Hà Nội, Hà Nội.

[5] Trần Đức Long - Nguyễn Đình Sang - Hoàng Quốc Toàn (2009), Bài tập giải tích tập II, Nhà xuất bản ĐHQG Hà Nội, Hà Nội.

[6] Nguyễn Đình Trí (chủ biên) - Tạ Văn Đĩnh Nguyễn Hồ Quỳnh (2001), Bài tập toán cao cấp tập hai, Nhà xuất bản Giáo dục, Hà Nội.

[7]. W.J.Kaczkor - M.T.Nowak (Người dịch: Đoàn Chi), Bài tập Giải tích 1, Nxb Đại học Sư phạm, 2003.

\section{Exploiting arithmetical properties related to numeral mathematical problems in student olympic exams}

Le Thieu Trang, Duong Thi Hong Hai

\section{Article info}

\section{Recieved:}

$2 / 5 / 2020$

Accepted:

$10 / 6 / 2020$

Keywords:

Sequence of numbers, limit, arithmetics, square number, student.

\begin{abstract}
The sequence of numbers is one of the subjects in the analytic program of the Mathematics Pedagogical University, it is a basic subject in teaching contents for the Olympic of Maths students in colleges and universities. The problems of sequence's number helps students understand more about the functions, the distribution rules of numbers, the properties of the infinitely small, infinitely great,... In this article, the author summarizes a number of problems related to the sequence of number to develop students' learning and research capacity.
\end{abstract}

